\title{
Syncope in Patients with Pacemakers
}

\author{
Richard Sutton
}

National Heart \& Lung Institute, Imperial College, London, UK

\begin{abstract}
Syncope in a pacemaker patient is a serious symptom but it is rarely due a pacemaker system malfunction. Syncope occurs in about $5 \%$ of patients paced for atrioventricular (AV) block in 5 years, 18\% in those paced for sinus node disease in 10 years, $20 \%$ of those paced for carotid sinus syndrome in 5 years and $5-55 \%$ of those older patients paced for vasovagal syncope in 2 years. The vastly different results in vasovagal syncope depend on the results of tilt testing, where those with negative tests approach results in pacing for AV block and those with a positive tilt test show no better results than with no pacemaker. The implication of tilt results is that a hypotensive tendency is clearly demonstrated by tilt positivity pointing to syncope recurrence with hypotension. This problem may be addressed by treatment with vasoconstrictor drugs in those who are suited or, more commonly, a reduction or cessation of hypotensive therapy in hypertensive patients. Other causes of syncope such as tachyarrhythmias are rare. The clinical approach to patients who report syncope is detailed.
\end{abstract}

\section{Keywords}

Syncope, pacemaker, pacemaker malfunction, atrioventricular block, sinus node disease, carotid sinus syndrome, vasovagal syncope, tilt testing

Disclosure: The author has no relevant conflicts of interest.

Received: 21 August 2015 Accepted: 3 November 2015 Citation: Arrhythmia \& Electrophysiology Review 2015;4(3):189-92. Access at: www.AERjournal.com Correspondence: Richard Sutton DSC, Emeritus Professor of Clinical Cardiology, ICCH Building, St Mary's Hospital Campus, 59-61 North Wharf Road, London W2 1LA, UK. E: r.sutton@imperial.ac.uk

Syncope in a patient with a pacemaker commands urgent action to ascertain its cause and provide appropriate treatment. This is a well accepted statement but the field has evolved in recent years and, strangely, has received little attention.

Many considerations bear on this issue. First, syncope in pacemaker patients is not common but may be more so than generally considered. The lack of frequency may be attributed to better technology and greater expertise amongst practitioners but it should be modulated by the fact that more patients today receive pacemakers for other indications than syncope, rendering them unlikely to sustain syncope even if their pacemaker system fails. Second, we now live in an era of telemedicine where remote monitoring not only provides an opportunity to identify technological or arrhythmic causes of syncope in a pacemaker patient but also a chance for the patient very readily to report such an episode to hospital carers. ${ }^{1}$ Third, more patients today receive pacemakers for reflex syncope where the device is not expected to achieve the results in syncope prevention that pertain in atrioventricular block (AVB). ${ }^{2,3}$ Included with this aspect of syncope in pacemaker patients must also now be the realisation that syncope may be reflex in the largest group of patients worldwide that receive pacemakers, those with sino-atrial node disease. ${ }^{4}$

It is in these contexts that a review of this serious clinical problem is due.

\section{Where We Were}

In the late 1980s and early 1990s, we had already achieved a high standard of implant techniques and devices were well constructed with rare failure. ${ }^{2,3}$ Langenfeld et al. found a $3.5 \%$ recurrence of syncope in AVB (Second and Third Degree) in 5 years; a question of reflex syncope did not arise. ${ }^{2}$
However, by 1991, Pavlovic et al., in a detailed analysis of $46 \mathrm{VVI}-$ paced patients with recurrent syncope, found only two with exit block and an additional patient with sensing failure, which was unlikely to have been the cause of syncope. ${ }^{3}$ Thus, $4.3 \%$ had pacing failure as the cause of syncope, while at the same time $8.6 \%$ had orthostatic hypotension and $36.9 \%$ were tilt positive. These tilt findings were invoked as explaining the syncope sustained by these patients but no explanation was found in another $30.4 \%$. They concluded that reflex syncope may be the most common cause of recurrent syncope in paced patients, with pacing hardware failure being quite rare.

This theme was reiterated by Sgarbossa et al. in a large series of 507 sick sinus syndrome patients from the Cleveland Clinic. ${ }^{4}$ In $62 \pm 38$ months of follow-up, they found syncope recurrence in $3 \%$ at 1 year, $8 \%$ at 5 years and $13 \%$ predicted at 10 years. Their analysis of the causes of syncope indicated lead or pacemaker failure in $6.5 \%$, vasovagal in $18 \%$, orthostatic hypotension in $25.5 \%$, unexplained in $29.5 \%$, atrial tachyarrhythmias in $11.5 \%$ and ventricular tachyarrhythmias in $5 \%$. They concluded that autonomic disturbances were the main contributors to syncope recurrence and pacing hardware failure was uncommon. Such conclusion was reached at a time when syncope in sick sinus syndrome was considered to be sinus arrest without effective escape mechanism rather than reflex in origin. However, Sgarbossa et al. noted that syncope prior to implant was the only reliable predictor of syncope post-implant.

Helguera et al. focused on endocardial lead malfunction with a broad definition including exit block in 1,474 patients from the Cleveland Clinic. ${ }^{5}$ In $33 \pm 32$ months follow-up, only 54 patients had lead malfunction and $37 \%$ had either syncope in 7 or pre-syncope 
in 13 patients. These symptomatic lead malfunctions were more common in ventricular leads and in those who had severe symptoms pre-implant. No deaths were attributed to lead malfunction. The authors recommended closest attention to those patients with severe presenting symptoms and pacemaker dependency.

A PubMed search shows that little has been published on these issues between the mid-1990s and the recent past.

\section{Where We Are Now}

New information on patients with sick sinus syndrome emerged from the DANPACE study, in which 1,415 patients were followed for 5.4 years. ${ }^{6}$ Syncope occurred in $17.5 \%$ of patients after pacing; of those with AAIR pacing, $19 \%$ had syncope whereas the figure was $15.8 \%$ in DDDR-paced patients. Predictors for syncope were: for age 0-39 years, hazard ratio (HR) 2.9; $>80$ years, HR 1.4; and syncope pre-implant, HR 1.8. Patients with syncope after pacing suffered a higher mortality (HR 1.6). The authors of this highly regarded trial concluded that syncope after pacing in sick sinus syndrome is common, carries an increased mortality and is multifactorial. It would appear that reflex syncope may have been an important factor because syncope in the general population and in their paced group share a bimodal distribution curve with respect to age. Given the longer follow-up in these patients, they have confirmed the earlier findings in much smaller populations. The increased mortality raises some anxiety, but may be explained by comorbidities rather than by a direct effect of syncope itself.

It is well known that vasovagal syncope recurs and risk of recurrence increases with more historical attacks, approaching $50 \%$ after six lifetime events. ' It is therefore likely that a patient who has experienced many attacks in the past will have recurrences after pacing, even if the clear indication for pacing was not reflex syncope. Further, if the indication for pacing is reflex syncope, recurrence is highly likely. Much more has been learned recently in the latter group of patients. It is also necessary to restate, in this context, that reflex syncope has two components, bradycardia/asystole and vasodepression. The former of these may be well treated by a pacemaker but vasodepression is uninfluenced by pacing. Thus, the blood pressure may fall profoundly with a maintained heart rate by a pacemaker.

The ISSUE-3 study was to the first to demonstrate that pacing is significantly effective in older vasovagal syncope patients. ${ }^{8}$ However, the results were tempered by the recurrence of syncope within 2 years of $25 \%$ in those paced versus $57 \%$ in those unpaced $(P<0.04)$. Reassurance was garnered from the nearly $18 \%$ syncope recurrence in sinus node disease reported from Denmark. ${ }^{6}$ Once the ISSUE-3 randomised controlled trial data were combined with the ISSUE-3 registry including patients (or physicians on their behalf) who refused pacing or the concept of randomisation at the point of randomisation, the importance of the tilt result was seen to be in terms of the outcome.? Tilt-negative patients (with implantable loop recorder (ILR) showing asystole in a spontaneous attack and otherwise clinically identical to those who were tilt positive with the same ILR findings) did well with pacing - $5 \%$ recurrence of syncope in 21 months. Tilt-positive patients did no better than if they had no pacemaker, with $55 \%$ recurrence rate of syncope. ${ }^{9}$

This finding at first appeared counter-intuitive, but analysis of the reported results of tilt testing over 28 years since the test was introduced for diagnosis of syncope pointed to an explanation..$^{10,11}$
The hypothesis suggests that tilt testing merely reveals a hypotensive or vasodepressive tendency and no longer should be termed diagnostic because this tendency is hidden by severe cardioinhibition. So a tilt-positive patient with severe cardioinhibition will not readily reveal accompanying vasodepression. Once paced, the pacemaker is expected to prevent cardioinhibition but cannot combat vasodepression, rendering recurrence of syncope likely. The argument was strengthened by the literature on carotid sinus syndrome (CSS), a similar reflex syncope, where those CSS patients with a positive tilt test had 2.7 times the recurrence rate of those who were tilt negative. ${ }^{12}$ These data have been further supported by a later report from the same group, with more patients showing very similar findings. ${ }^{13}$ The Italian SUP-2 study, including 10 Italian centres, recently reported a decision algorithm for older patients presenting clinically likely reflex syncope. ${ }^{14}$ The decision-tree demanded carotid sinus massage (CSM) according to the 'method of symptoms' as a first test. Those who had positive CSM were paced, those negative proceeded to a tilt test and, if positive with asystole, were also paced. Those who were tilt negative proceeded to ILR and, if positive with asystole in a spontaneous attack, were then paced. This algorithm permitted assessment of those who were tilt negative and ILR positive and it has been shown separately from the ISSUE-3 study that these patients do well with pacing with a very low syncope recurrence rate in 13 months of follow-up.

Thus, in reflex syncope, both vasovagal and CSS, recurrence of syncope can be predicted after pacing by the tilt test result. The hypothesis also addressed the frequent question in this age group of hypertension and its treatment. ${ }^{11}$ Hypertension in these patients is often aggressively treated, producing side effects of episodes of orthostatic hypotension. It is considered likely that some of these patients, as a result, start to experience vasovagal syncope or it recurs (patients having sustained a few attacks in youth). Great care in administering hypotensive medication must now be the norm for these patients and randomised controlled trials are indicated. This group of patients should be considered at risk for syncope recurrence similarly to those who are tilt positive as in the ISSUE-38,9 and SUP- $2^{14}$ studies.

Syncope in paced patients has become much more clear now. It is either rarely due to pacing hardware malfunction or much more commonly due to reflex syncope occurring spontaneously or favoured by hypotensive treatment of hypertension.

\section{How should Paced Patients who Report Syncope be Approached?}

Syncope in the paced patient must be regarded as a serious symptom and be promptly investigated. The first consideration should be: was the correct diagnosis of the original syncope made? It is possible that syncope was reflex rather than conduction tissue disease. The second thought should be: are drugs playing a part? If the patient is hypertensive and on hypotensive treatment, this may be the cause of orthostatic hypotension or a rekindling of vasovagal syncope of youth. The third matter to consider is whether the pacing mode is correct. Patients with VVI pacing may have bouts of retrograde AV conduction that can precipitate syncope or, rarely, AAI mode has been selected for a patient with AVB. The fourth matter for thought is whether a tachyarrhythmia could be responsible for recurrence of syncope. In this instance, telemonitoring is extremely valuable for defining the arrhythmia. In such cases, assessment of left ventricular function by echocardiography may be helpful in showing deterioration of function 
that might be compatible with ventricular arrhythmias. Also, in the less likely possibility that atrial fibrillation is responsible for syncope, echocardiographic assessment of left atrial size is indicated.

Patients paced for AVB presenting syncope represent the most likely to have a pacing hardware fault as they are likely to be pacemaker dependent. First, it is necessary to make sure that the device is appropriate for the implanting indication and then consider the possibility of a technical fault. Always, it must be borne in mind that such faults may be difficult to identify in the clinic. In this instance, telemonitoring is much more effective. ${ }^{1}$

Early after implantation, the technical fault might be lead displacement or perforation or incomplete connection at the lead-pulse generator interface. Exit block may present early but also after the first month of implantation. The term 'exit block' implies that there is an excessive reaction by the endocardium at the site of the electrode, which has raised the stimulation threshold to a level above the output of the pacemaker. The pacemaker then fails to capture the heart. Some devices now measure the stimulation threshold and adjust output to address this problem. Later problems include lead insulation failure causing failure to capture heralded by low lead impedance, lead conductor fracture heralded by high lead impedance and possibly by oversensing. While these late problems are mostly lead related there could also be a pulse generator problem including normal or early battery depletion. Again, in all of these problems, telemonitoring is very important and must now be considered the standard of care. ${ }^{15}$

In patients with indications for pacing other than AVB, technical faults are also possible and must be excluded - telemonitoring is also ideal for this. After such a fault is considered very unlikely, a tilt test is advised to reveal a possible hypotensive tendency. If present, this may be the explanation for the patient's problems but if absent hypotension could be considered unlikely. It should be borne in mind that a tilt test should no longer be taken as diagnostic but as a risk-ofsyncope-recurrence stratification tool. ${ }^{11}$

The patient needs to be seen and, if known to be pacemaker dependent, perhaps directly admitted to hospital (see Figure 1). In clinic, the usual assessments must be made, also including deep respiration and movement of the pulse generator to attempt to expose evidence of lead damage. A 12-lead ECG must be taken, which is something often not done in the pacemaker clinic. The pattern of depolarisation may have changed from that after implant, implying lead displacement. A chest X-ray may be needed and may show evidence of lead displacement, lead damage or twiddler's syndrome. The evidence from remote monitoring must be set against the data collected in the clinic. Is the pacing mode and programme correct? The initial assessment for syncope, as in the European Society of Cardiology Guidelines, ${ }^{16}$ should be undertaken as this may not have been done at implant. Last, the patient
Figure 1: Management of Patients with a Pacemaker Reporting Syncope

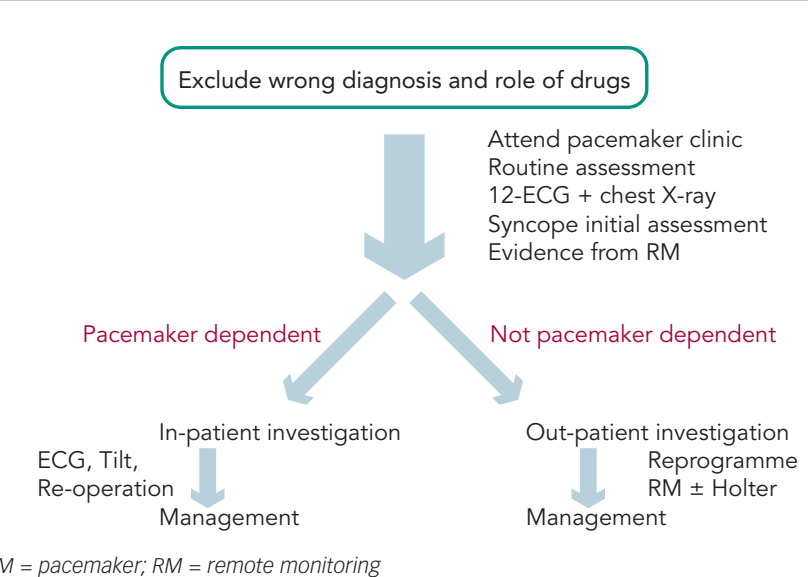

should be considered for admission to hospital - usually necessary for pacemaker-dependent patients, but unlikely to be necessary for patients not pacemaker dependent. For the non-dependent patient, remote monitoring should immediately be established if not already done. If remote monitoring is unavailable, prolonged Holter monitoring will often be required. Hospital admission is usually undertaken when a reoperation is needed rather than attempting therapy as a day case. It should be noted that diagnostic reoperation is now most unlikely to be needed because telemonitoring is so much more effective.

Patient management after diagnosis of the cause of syncope may be reoperation to correct the identified fault, lead repositioning, lead replacement, generator change or upgrading of the pacemaker system. In less serious problems, reprogramming may be sufficient. In reflex syncope cases, use of a rate-drop response type of pacemaker algorithm may help, especially making use of data from tilt testing to tune the program. A closed-loop pacemaker may be more effective than others in reflex syncope but no trial proof of this is currently available. For those who are tilt positive, attempting vasoconstrictor therapy with a drug such as midodrine may help. When it appears that sinus tachycardia is the trigger for syncope, antagonism of this by ivabradine or beta-blocker may help. These patients always need much reassurance because recurrence of pre-pacing symptoms does so much to undermine confidence.

In conclusion, syncope in a pacemaker patient is a serious symptom requiring action. It is quite rare for its explanation to be pacemaker hardware malfunction (around $5 \%$ of cases) and it is much more commonly due to reflex syncope involving vasodepression, which may be iatrogenic by excessive hypotensive therapy. Syncope in pacemaker patients is not as rare as often thought, occurring in $18 \%$ of sinus node disease patients in 10 years and $55 \%$ of tilt positive vasovagal patients in 2 years.
1. Varma N. Automatic home monitoring of patients with cardiac implantable electronic devices - the setting of a new standard. Europace 2013;15:Suppl 1

2. Langenfeld $\mathrm{H}$, Grimm W, Maisch B, et al. Course of symptoms and spontaneous ECG in pacemaker patients: a 5-year follow-up study. Pacing Clin Electrophysiol 1988;11:2198206.

3. Pavlovic SU, Kocovic D, Djordjevic M, et al. The etiology of syncope in pacemaker patients. Pacing Clin Electrophysiol 1991:14:2086-91.

4. Sgarbossa EB, Pinski SL, Jaeger FJ, et al. Incidence and predictors of syncope in paced patients with sick sinus syndrome. Pacing Clin Electrophysiol 1992;15:2055-60.
5. Helguera ME, Maloney JD, Fahy GJ, et al. Clinical presentation of endocardial lead malfunction. Am J Cardiol 1996:78:1297-9. Of endocardial lead malfunction. Am J Cardiol 1996;78:1297-9.
Chuen NK, Kirkfeldt RE, Andersen HR, et al. Syncope in paced patients with sick sinus syndrome from the DANPACE trial: incidence, predictors and prognostic implication. Heart 2014;100:842-7.

7. Rose MS, Koshman ML, Spreng S, et al. The relationship between health-related quality of life and the frequency of spells in pationts with syncope J Clin Epictiol 2000:53:1209-16.

- Brignol Mi Menozzic Moya A, et al Pacemaker therapy in patients with neurally-mediated syncope and documented asystole. Third international study on syncope of unknown etiology (ISSUE-3): a randomized trial. circulation
2012:125:2566-71.

9. Brignole $\mathrm{M}$, Donateo $\mathrm{P}$, Tomaino $\mathrm{M}$, et al. The benefit of pacemaker therapy in patients with presumed neurallymediated syncope and documented asystole is greater when tilt test is negative. An analysis from the third international study on syncope of uncertain etiology (ISSUE 3). Circ Arrhythm Electrophysiol 2014;7:10-6.

10. Kenny RA, Ingram A, Bayliss J, et al. Head-up tilt: a useful test for investigating unexplained syncope. Lancet 1986;1:1352-5.

11. Sutton $R$, Brignole $M$. Twenty-eight years of research permit reinterpretation of tilt-testing: hypotensive susceptibility rather than diagnosis. Eur Heart J 2014;35:2211-2. 


\section{Device Therapy}

12. Gaggioli G, Brignole M, Menozzi C, et al. Reappraisal of the vasodepressor reflex in carotid sinus syndrome. Am I Cardiol 1995;75:518-21.

13. Solari $\mathrm{D}$, Maggi R, Oddone $\mathrm{D}$, et al. Clinical context and outcome of carotid sinus symdrome diagnosed by means of the 'method of symptoms'. Europace 2014:16:928-34.
14. Brignole M, Ammirati F, Arabia F, et al. Assessment of a standardized algorithm for cardiac pacing in older patients affected by severe unpredictable reflex syncopes. Eur Heart 2015;36:1529-35.

15. Sutton R. Remote monitoring as a key innovation in the management of cardiac patients including those with implantable electronic devices. Europace 2013;15:13-i5. 16. Moya, A, Sutton R, Ammirati F, et al. The Task Force for the Diagnosis and Management of Syncope of the European Society of Cardiology (ESC). Guidelines for the diagnosis and management of syncope (version 2009). Eur Heart J 2009:30:2631-71. 